\title{
Three Vertex and Parallelograms in the Affine Plane: Similarity and Addition Abelian Groups of Similarly n-Vertexes in the Desargues Affine Plane
}

\author{
Orgest Zaka \\ Department of Mathematics, Faculty of Technical Science, University “Ismail QEMALI”of Vlora, Vlora, Albania
}

\section{Email address:}

gertizaka@yahoo.com

\section{To cite this article:}

Orgest Zaka. Three Vertex and Parallelograms in the Affine Plane: Similarity and Addition Abelian Groups of Similarly $n$-Vertexes in the Desargues Affine Plane. Mathematical Modelling and Applications. Vol. 3, No. 1, 2018, pp. 9-15. doi: 10.11648/j.mma.20180301.12

Received: May 14, 2017; Accepted: December 18, 2017; Published: January 8, 2018

\begin{abstract}
In this article will do a' concept generalization $n$-gon. By renouncing the metrics in much axiomatic geometry, the need arises for a new label to this concept. In this paper will use the meaning of $n$-vertexes. As you know in affine and projective plane simply set of points, blocks and incidence relation, which is argued in [1], [2], [3]. In this paper will focus on affine plane. Will describe the meaning of the similarity $n$-vertexes. Will determine the addition of similar three-vertexes in Desargues affine plane, which is argued in [1], [2], [3], and show that this set of three-vertexes forms an commutative group associated with additions of three-vertexes. At the end of this paperare making a generalization of the meeting of similarity $n$ vertexes in Desargues affine plane, also here it turns out to have a commutative group, associated with additions of similarity $n$-vertexes.
\end{abstract}

Keyword: $n$-vertexes, Desargues Affine Plane, Similarity of $n$-Vertexes, Abelian Group

\section{Introduction}

In Euclidian geometry use the term three-angle and non three-vertex, this because the fact that the Euclidean geometry think of associated with metrics, which are argued in [4], [6], [7]. In this paper will use the "three-Vertex" term, by renouncing the metric. Will generalize so its own meaning in the Euclidean case. With the help of parallelism [1], [2], [3] will give meaning of similarity and will see that have a generalization of the similarity of the figures in the Euclidean plane. By following the logic of additions of points in a line of Desargues affine plane submitted to [3], herewill show that analogously this meaning may also extend to the addition of similarity three-vertex in Desargues affine plane, moreover extend this concept for the similarity $n$-vertexes to the Desargues affine plane.

The aim is to see if the move to three-vertexes as well as to $n$-vertexes has the group's properties, which are arguing that the best in [5], [8], [9].

\section{2. $n$-Vertexes in Affine Plane and Their Similarity}

\subsection{3-Vertexes and Their Similarity}

Let's have the affine plane $\mathcal{A}=(\mathcal{P}, \mathcal{L}, \mathcal{I})$.

Definition 2.1.1 Three-Vertex will called an ordered trio of non-collinear points $(A, B, C)$ in an affine plane.

Definition 2.1.2 Two three-vertexes $\left(A_{1}, B_{l}, C_{l}\right)$ and $\left(A_{2}, B_{2}, C_{2}\right)$ will call similar if they meet conditions: $A_{1} B_{1} / / A_{2} B_{2} ; A_{1} C_{1} / / A_{2} C_{2}$ and $B_{1} C_{1} / / B_{2} C_{2}$

Example 2.1.1 In affine plane of the second order have the similar three-vertices (Figure 1):

$$
\begin{aligned}
& (A, D, C) \approx(B, C, D): \text { because, } \\
& A D\|B C ; D C\| C D ; A C \| B D \\
& (A, B, D) \approx(C, D, B): \text { because, } \\
& A B\|C D ; B D\| D B ; A D \| C B
\end{aligned}
$$




$$
(A, B, D) \approx(D, C, A): \text { because, }
$$$$
A B\|D C ; B D\| C A ; A D \| D A
$$

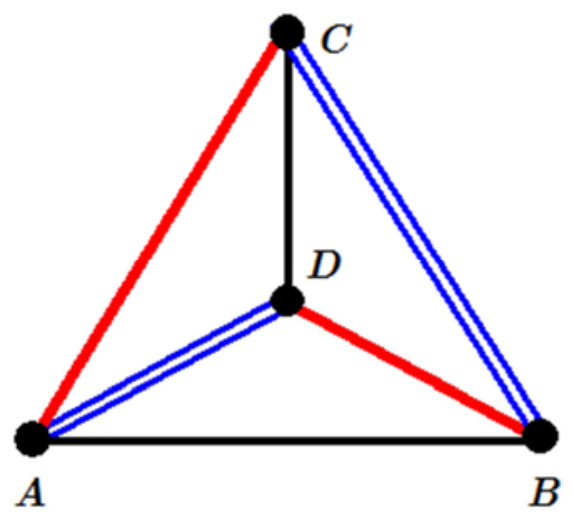

Figure 1. The similar three-vertexes in affine plane of order 2.

Example 2.1.2: In the third order affine plane.

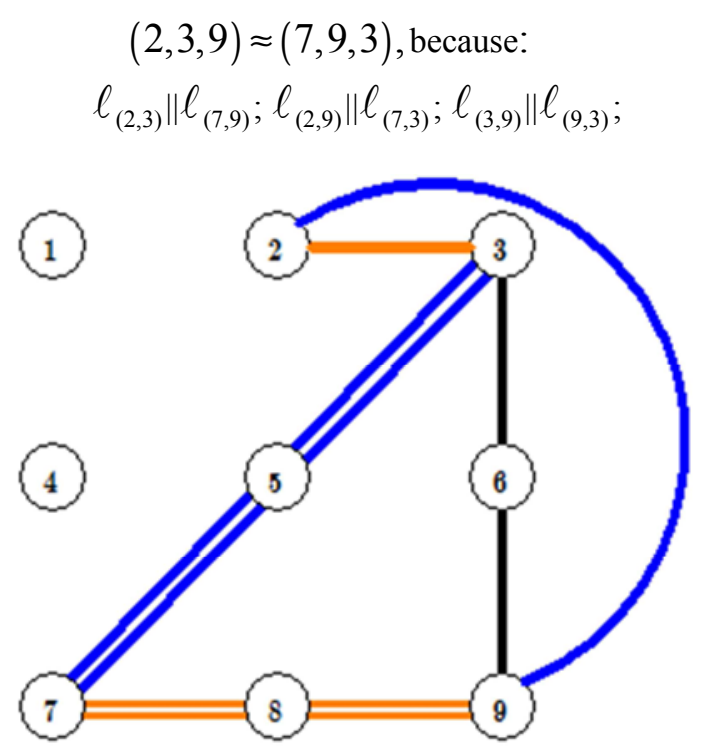

Figure 2. Two similar three-vertexes in affine plane of order 3.

Proposition 2.1.1: The similarity of the three-vertexes is equivalence relation.

Proof: 1) It is clear that every three-vertexes $(A, B, C)$ is similar to yourself.

$$
(A, B, C) \approx(A, B, C)
$$

2) If three-vertexes $\left(A_{1}, B_{1}, C_{1}\right) \approx\left(A_{2}, B_{2}, C_{2}\right)$, are similar then also three-vertexes $\left(A_{2}, B_{2}, C_{2}\right) \approx\left(A_{1}, B_{1}, C_{l}\right)$, are similarity since from:

$$
\begin{aligned}
& A_{1} B_{1} / / A_{2} B_{2} ; A_{1} C_{1} / / A_{2} C_{2} ; B_{1} C_{1} / / B_{2} C_{2} \Longrightarrow \\
& \Rightarrow A_{2} B_{2} / / A_{1} B_{1} ; A_{2} C_{2} / / A_{1} C_{1} ; B_{2} C_{2} / / B_{1} C_{1} .
\end{aligned}
$$

3) If $\left(A_{1}, B_{1}, C_{1}\right) \approx\left(A_{2}, B_{2}, C_{2}\right)$, and three-vertexes $\left(A_{2}, B_{2}, C_{2}\right) \approx$ $\left(A_{3}, B_{3}, C_{3}\right)$ then have to $\left(A_{1}, B_{1}, C_{1}\right) \approx\left(A_{3}, B_{3}, C_{3}\right)$, because parallelism in the affine plane is equivalence relation, which is described in [2], [3], [4].

So would have to:

$$
A_{1} B_{1} / / A_{2} B_{2} ; A_{1} C_{l} / / A_{2} C_{2} ; B_{l} C_{l} / / B_{2} C_{2}
$$

and

$$
A_{2} B_{2} / / A_{3} B_{3} ; A_{2} C_{2} / / A_{3} C_{3} ; B_{2} C_{2} / / B_{3} C_{3}
$$

since the parallelism in the affine plane is equivalence relation then will have to:

$$
\begin{aligned}
& A_{1} B_{1} / / A_{2} B_{2} \text { and } A_{2} B_{2} / / A_{3} B_{3} \Longrightarrow A_{1} B_{1} / / A_{3} B_{3} ; \\
& A_{1} C_{1} / / A_{2} C_{2} \text { and } A_{2} C_{2} / / A_{3} C_{3} \Rightarrow A_{1} C_{1} / / A_{3} C_{3} ; \\
& B_{1} C_{1} / / B_{2} C_{2} \text { and } B_{2} C_{2} / / B_{3} C_{3} \Rightarrow B_{1} C_{1} / / B_{3} C_{3} .
\end{aligned}
$$

Well,

$$
\left(A_{1}, B_{1}, C_{1}\right) \approx\left(A_{3}, B_{3}, C_{3}\right)
$$

\subsection{4-Vertexes}

Definition 2.2.1: In affine plane $\mathcal{A}$, a set of four-point three out of three not-collineary will call 4-vertexes.

Definition 2.2.2: Two 4-vertexes $A B C D$ and $A$ ' $B$ ' $C$ ' $D$ ' will call similar only if have the following parallels: $\mathrm{AB}\left\|\mathrm{A}^{\prime} \mathrm{B}^{\prime}, \mathrm{BC}\right\| \mathrm{B}^{\prime} \mathrm{C}^{\prime}, \mathrm{CD} \| \mathrm{C}^{\prime} \mathrm{D}^{\prime}$ and $\mathrm{DA} \| \mathrm{D}^{\prime} \mathrm{A}^{\prime}$.

\subsection{Parallelograms}

Definition 2.1.3: Parallelogram will call the ordered quartet of points $(A, B, C, D)$ from $\mathcal{P}$, that meets the conditions: $A B / / C D$ and $B C / / A D$ the lines $A C$ and $B D$ are called the diagonal of parallelogram.

Example 2.2.1: In affine plane of the second order (Figure 3. a.) have the following parallelogram:

$(\mathrm{A}, \mathrm{D}, \mathrm{B}, \mathrm{C})$ with the diagonal $\mathrm{AB}$ and $\mathrm{DC}$ (Figure 3. b);

$(\mathrm{A}, \mathrm{B}, \mathrm{D}, \mathrm{C})$ with the diagonal $\mathrm{AD}$ and $\mathrm{BC}$ (Figure 3. c); (A,B,C,D) with the diagonal $\mathrm{AC}$ and $\mathrm{BD}$ (Figure 3. d).

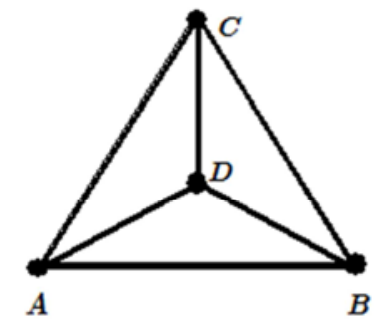

(a)

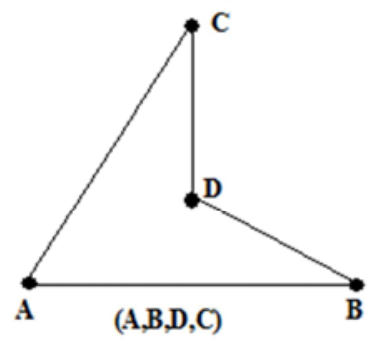

(c)

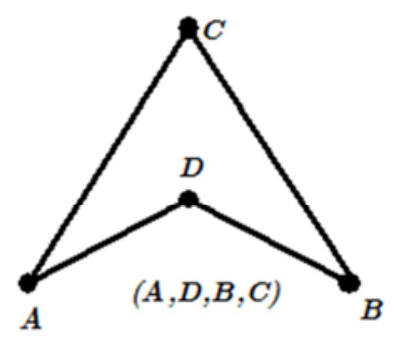

(b)

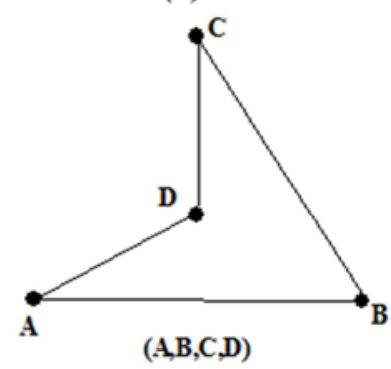

(dI)
Figure 3. 4-parallelograms in the affine plane of order 2.

From the definition of parallelogram and the fact that parallelism is the equivalence relation is evident this Proposition:

Proposition 2.2.1: If you have two similar 4-vertexes, where each is parallelogram then another 4-vertexes will be parallelogram.

\section{4. $n$-Vertexes}

Definition 2.4.1: In affine plane $\mathcal{A}$, a set of n-points non- 
collinearly three out of three will call n-vertex.

Definition 2.4.2: Two n-vertexes $\left(A_{1} A_{2} \ldots A_{n}\right)$ and $\left(B_{1} B_{2} \ldots B_{n}\right)$ will call similar just if have the following parallelisms:

$$
\begin{gathered}
\mathbf{A}_{\mathbf{i}} \mathbf{A}_{\mathbf{j}} \| \mathbf{B}_{\mathbf{i}} \mathbf{B}_{\mathbf{j}}, \\
\forall(i, j) \in\{(1,1), \ldots,(1, n) ;(2,1), \ldots,(2, n) ; \ldots ;(n, 1), \ldots,(n, n)\} .
\end{gathered}
$$

\section{The Addition of Similarity Three- Vertexes in the Desargues Affine Plane}

Let's have two similarity three-vertexes $\left(\mathrm{A}_{1}, \mathrm{~A}_{2}, \mathrm{~A}_{3}\right)$ and $\left(\mathrm{B}_{1}, \mathrm{~B}_{2}, \mathrm{~B}_{3}\right)$ in the Desargues affine plane $\mathcal{A}_{D}=(\mathcal{P}, \mathcal{L}, \mathcal{I})$. Constructed the lines $\mathrm{A}_{1} \mathrm{~B}_{1}, \mathrm{~A}_{2} \mathrm{~B}_{2}, \mathrm{~A}_{3} \mathrm{~B}_{3}$, since are in Desargues affine plane and the similarity of three-vertexes have to: $A_{1} A_{2}\left\|B_{1} B_{2} ; \quad A_{2} A_{3}\right\| B_{2} B_{3} ; \quad A_{1} A_{3} \| B_{1} B_{3} \Rightarrow$ the lines $A_{1} B_{1}, A_{2} B_{2}$ and $A_{3} B_{3}$, or will be parallel or will cross the on a single point. Receive now a point $\mathrm{O}_{1} \in \mathrm{A}_{1} \mathrm{~B}_{1}$, and find points
$\mathrm{O}_{2}$ and $\mathrm{O}_{3}$ how:

$$
\boldsymbol{O}_{2}=A_{2} B_{2} \cap \ell_{A_{1} A_{2}}^{O_{1}} \text { and } O_{3}=A_{3} B_{3} \cap \ell_{A_{1} A_{3}}^{O_{1}}
$$

So have obtained thus three-vertexes $\left(\mathrm{O}_{1}, \mathrm{O}_{2}, \mathrm{O}_{3}\right)$, (points $\mathrm{O}_{1}, \mathrm{O}_{2}$ and $\mathrm{O}_{3}$ are non-collinearly, because from construction this three-vertexes will be similar with three-vertexes $\left.\left(A_{1}, A_{2}, A_{3}\right)\right)$ where $O_{1} \in A_{1} B_{1}, O_{2} \in A_{2} B_{2}$ and $O_{3} \in A_{3} B_{3}$. This three-vertex called 'zero' three-vertex. So have three lines, to which each have its zero point. Now just as to [3], additions of the points of each line based on the algorithm of additions of points in a line in Desargues affine plans, and take:

$$
C_{1}=A_{1}+B_{1}, C_{2}=A_{2}+B_{2}, C_{3}=A_{3}+B_{3} \text {. }
$$

Definition 3.1: The addition of two similarity threevertexes $\left(\mathbf{A}_{\mathbf{1}}, \mathbf{A}_{\mathbf{2}}, \mathbf{A}_{\mathbf{3}}\right)$ and $\left(\mathbf{B}_{\mathbf{1}}, \mathbf{B}_{\mathbf{2}}, \mathbf{B}_{\mathbf{3}}\right)$,called three-vertexes $\left(\mathbf{C}_{\mathbf{1}}, \mathbf{C}_{\mathbf{2}}, \mathbf{C}_{\mathbf{3}}\right)$, where the points (vertexes) $\mathbf{C}_{\mathbf{1}}, \mathbf{C}_{\mathbf{2}}, \mathbf{C}_{\mathbf{3}}$. They found according to equation (1) (Figure 4).

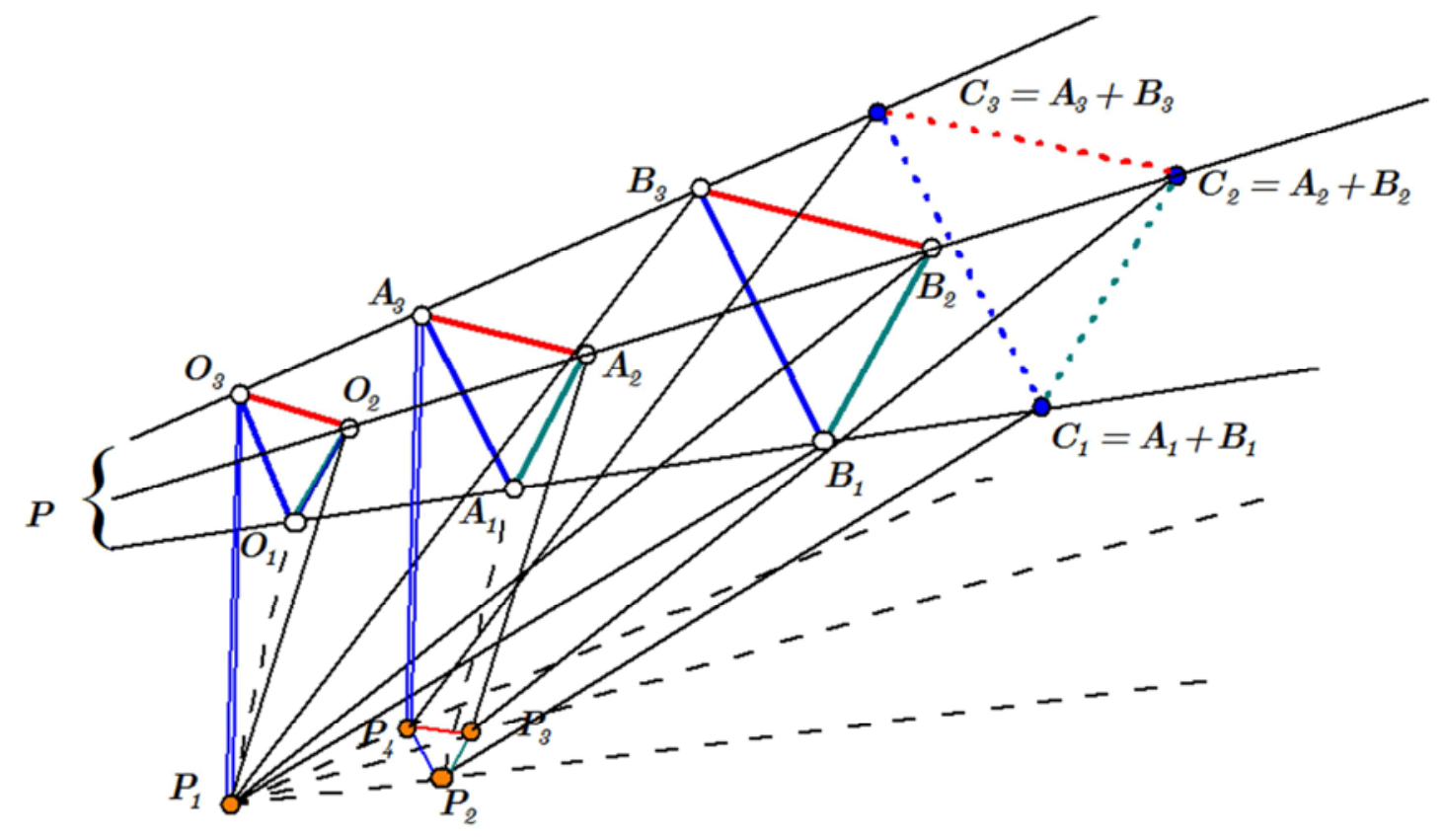

Figure 4. The Addition of two similarity three-Vertexes in the Desargues Affine Plane.

From construction of three-vertexes as the addition of two similar three-vertexes have evident this Proposition:

Proposition 3.1 Three-vertexes that obtained as the sum of two similar three-vertexes $\left(A_{1}, A_{2}, A_{3}\right)$ and $\left(B_{1}, B_{2}, B_{3}\right)$, it is similar to the first two.

Well

$$
\left(\mathrm{A}_{1}+\mathrm{B}_{1}, \mathrm{~A}_{2}+\mathrm{B}_{2}, \mathrm{~A}_{3}+\mathrm{B}_{3}\right) \approx\left(\mathrm{A}_{1}, \mathrm{~A}_{2}, \mathrm{~A}_{3}\right)
$$

and

$$
\left(\mathrm{A}_{1}+\mathrm{B}_{1}, \mathrm{~A}_{2}+\mathrm{B}_{2}, \mathrm{~A}_{3}+\mathrm{B}_{3}\right) \approx\left(\mathrm{B}_{1}, \mathrm{~B}_{2}, \mathrm{~B}_{3}\right)
$$

Proposition 3.2 The additions of non-similarity threevertexes it may not be a three-vertexes.
Proof: If renounce above from addition algorithm of the similar three-vertexes. In the same logic, are additions together two of whatever three-vertexes. Let's have two whatever three-vertexes $\left(\mathrm{A}_{1}, \mathrm{~A}_{2}, \mathrm{~A}_{3}\right)$ and $\left(\mathrm{B}_{1}, \mathrm{~B}_{2}, \mathrm{~B}_{3}\right)$ in the affine plane $\mathcal{A}=(\mathcal{P}, \mathcal{L}, \mathcal{I})$. Construct the line $\mathrm{A}_{1} \mathrm{~B}_{1}, \mathrm{~A}_{2} \mathrm{~B}_{2}$ and $\mathrm{A}_{3} \mathrm{~B}_{3}$. Get a whatever three-vertexes $\left(\mathrm{O}_{1}, \mathrm{O}_{2}, \mathrm{O}_{3}\right)$ (the points $\mathrm{O}_{1}, \mathrm{O}_{2}$ and $\mathrm{O}_{3}$ are non-collinearly) where $\mathrm{O}_{1} \in \mathrm{A}_{1} \mathrm{~B}_{1}$, $\mathrm{O}_{2} \in \mathrm{A}_{2} \mathrm{~B}_{2}$ and $\mathrm{O}_{3} \in \mathrm{A}_{3} \mathrm{~B}_{3}$. This three-vertexes called the 'zero' three-vertex. So have three lines, where, in each line have hers zero point. Now just as to [3], the addition points of every line based on the addition algorithm given to [3], and take: $\mathrm{C}_{1}=\mathrm{A}_{1}+\mathrm{B}_{1}, \mathrm{C}_{2}=\mathrm{A}_{2}+\mathrm{B}_{2}$ and $\mathrm{C}_{3}=\mathrm{A}_{3}+\mathrm{B}_{3}$. 


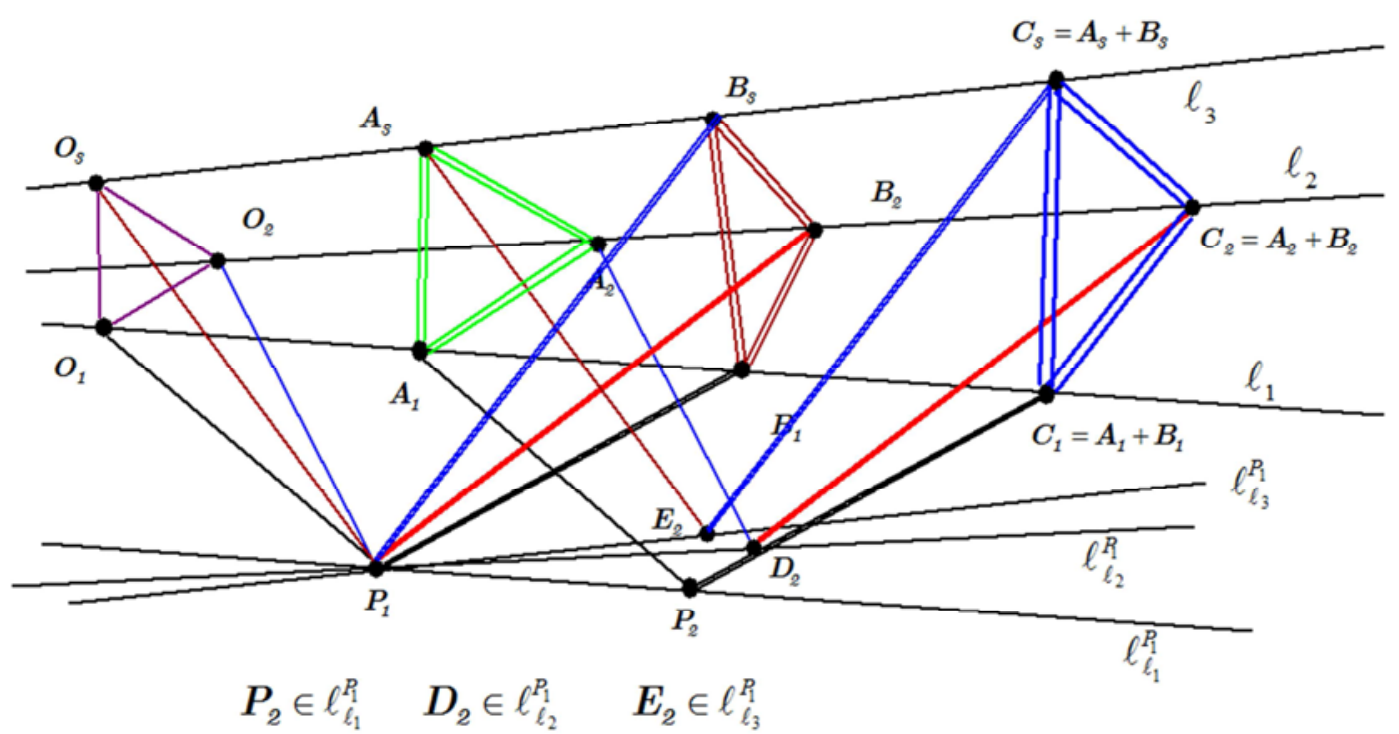

Figure 5. The Addition of two non-similarity three-Vertexes in the Desargues Affine Plane is a three-Vertex.

Defined in this way, it seems as if does not have a contradiction. But the veracity of this Proposition are presenting with the help of a simple anti-example, shown in the following figure.

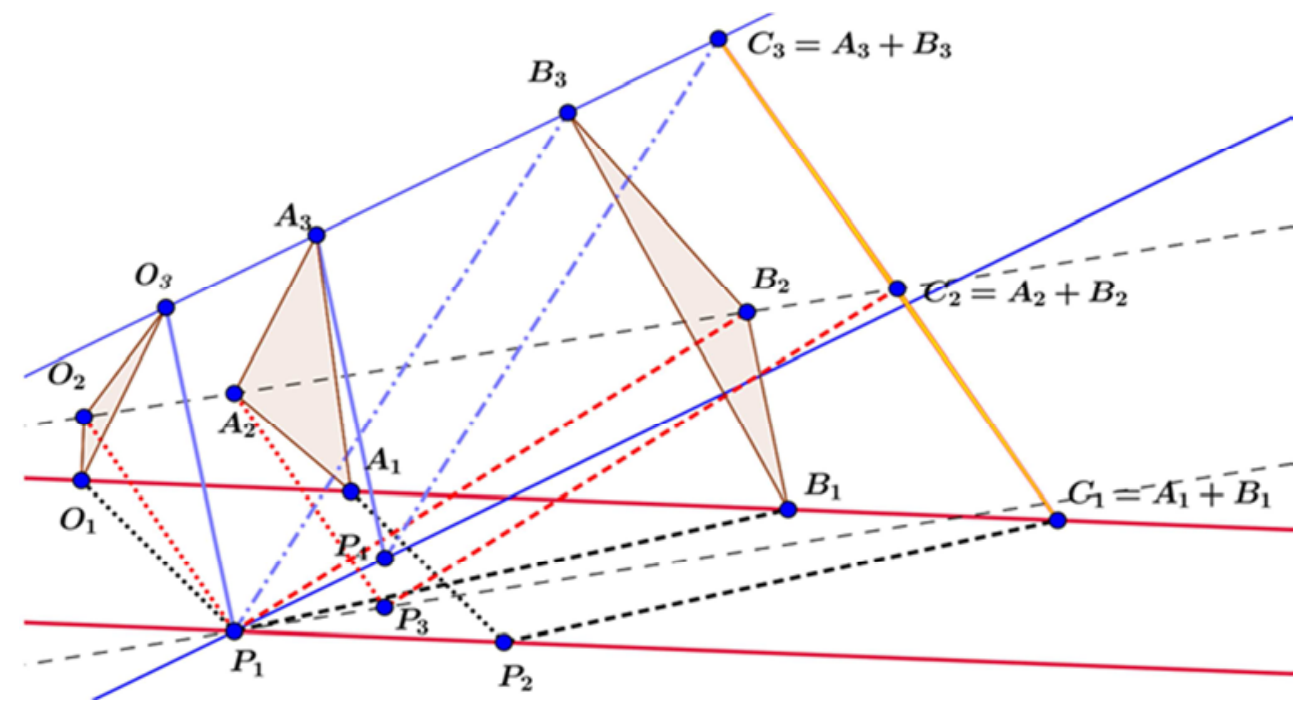

Figure 6. The Addition of two non-similarity three-Vertexes in the Desargues Affine Plane is not a three-Vertex.

Remark 3.1: By following the addition algorithms for points in a line of Desargues affine plane, is sufficient to get only an auxiliary point $\mathrm{P}_{1}$, for this obedient from [3], for three sums can either take one three-vertexes $\left(\mathrm{P}_{1}, \mathrm{P}_{2}, \mathrm{P}_{3}\right)$, wherein each point of three-vertexes be auxiliary point for the relevant sum.

Remark 3.2: Marked the set of three-vertexes in the Desargues affine plans with symbol $\mathcal{T}^{\mathcal{D} \text {.Aff }}$.

Remark 3.3: Marked the set of similarity three-vertexes in the Desargues affine plans with symbol $\mathcal{T}_{\approx}^{\mathcal{D} . A f f}$.

It is clear that: $\mathcal{T}_{\approx}^{\mathcal{D} . A f f} \subset \mathcal{T}^{\mathcal{D} \cdot A f f}$.

Let us be $\left(\boldsymbol{A}_{1}, \boldsymbol{A}_{2}, \boldsymbol{A}_{3}\right)$ and $\left(\boldsymbol{B}_{1}, \boldsymbol{B}_{2}, \boldsymbol{B}_{3}\right)$ two whatsoever three-vertexes in the set $\mathcal{T}_{\approx}^{\mathcal{D}}$.Aff . I associate pairs

$$
\left[\left(A_{1}, A_{2}, A_{3}\right),\left(B_{1}, B_{2}, B_{3}\right)\right] \in \mathcal{T}_{\approx}^{\mathcal{D} . A f f} \times \mathcal{T}_{\approx}^{\mathcal{D} . A f f}
$$

three-vertex $\left(C_{1}, C_{2}, C_{3}\right) \in \mathcal{T}_{\approx}^{\mathcal{D} . A f f}$, that the vertexes are determines with algorithm in [3]. According to the preceding Theorems, three-vertexes $\left(\boldsymbol{C}_{1}, \boldsymbol{C}_{2}, \boldsymbol{C}_{3}\right)$ is determined in single mode by [3].

Thus obtain an application

$$
\mathcal{T}_{\approx}^{\mathcal{D} \cdot A f f} \times \mathcal{T}_{\approx}^{\mathcal{D} \cdot A f f} \rightarrow \mathcal{T}_{\approx}^{\mathcal{D} \cdot A f f}
$$

Definition 3.2: In the above conditions, application

$$
+: \mathcal{T}_{\approx}^{\mathcal{D} . A f f} \times \mathcal{T}_{\approx}^{\mathcal{D} . A f f} \rightarrow \mathcal{T}_{\approx}^{\mathcal{D} . A f f}
$$

defined by 


$$
\begin{gathered}
{\left[\left(A_{1}, A_{2}, A_{3}\right),\left(B_{1}, B_{2}, B_{3}\right)\right] \mapsto\left(C_{1}, C_{2}, C_{3}\right)} \\
\forall\left[\left(A_{1}, A_{2}, A_{3}\right),\left(B_{1}, B_{2}, B_{3}\right)\right] \in \mathcal{T}_{\approx}^{\mathcal{D} . A f f} \times \mathcal{T}_{\approx}^{\mathcal{D} . A f f} .
\end{gathered}
$$

The addition in $\mathcal{T}_{\approx}^{\mathcal{D} . A f f}$ according to this Definitions, can write

$$
\begin{aligned}
& \forall\left(A_{1}, A_{2}, A_{3}\right),\left(B_{1}, B_{2}, B_{3}\right) \in \mathcal{T}_{\approx}^{\mathcal{D} . A f f}, \\
& \text { 1. } P_{1} \notin A_{1} B_{1}, A_{2} B_{2}, A_{3} B_{3}, \\
& \text { 2. } \ell_{A_{1} B_{1}}^{P_{1}} \cap \ell_{O_{1} P_{1}}^{A_{1}}=P_{2}, \\
& \text { 3. } \ell_{P_{1} B_{1}}^{P_{2}} \cap A_{1} B_{1}=C_{1} . \\
& \text { 4. } \ell_{A_{2} B_{2}}^{P_{1}} \cap \ell_{O_{2} P_{1}}^{A_{2}}=P_{3}, \\
& \text { 5. } \ell_{P_{1} B_{2}}^{P_{3}} \cap A_{2} B_{2}=C_{2} . \\
& \text { 6. } \ell_{A_{3} B_{3}}^{P_{1}} \cap \ell_{O_{3} P_{1}}^{A_{3}}=P_{4}, \\
& \text { 7. } \ell_{P_{1} B_{3}}^{P_{2}} \cap A_{3} B_{3}=C_{3} . \\
& \Leftrightarrow\left(A_{1}, A_{2}, A_{3}\right)+\left(B_{1}, B_{2}, B_{3}\right)=\left(C_{1}, C_{2}, C_{3}\right) .
\end{aligned}
$$

Theorem 3.1: For every two three-vertexes $\left(A_{1}, A_{2}, A_{3}\right)$, $\left(\boldsymbol{B}_{1}, \boldsymbol{B}_{2}, \boldsymbol{B}_{3}\right) \in \mathcal{T}_{\approx}^{\mathcal{D} . A f f}$, algorithm (2) determines the single three-vertexes $\left(\boldsymbol{C}_{1}, \boldsymbol{C}_{2}, \boldsymbol{C}_{3}\right) \in \mathcal{T}_{\approx}^{\mathcal{D} \text {.Aff }}$, which does not depend on the choice of hers auxiliary point $\boldsymbol{P}_{1}$.

Proof: From Theorem 2.1, in [3], have to addition of two points in a line of Desargues affine plane does not depend on the choice of hers auxiliary point. For this reason keep as auxiliary points for addition of pairs points, the auxiliary point $\mathrm{P}_{1}$.

From Theorem 3.1, appears immediately true this

Proposition 3.3: Additions of three-vertexes in $\mathcal{T}_{\approx}^{\mathcal{D} . A f f}$ there are element zero the three-vertexes $\left(\mathrm{O}_{1}, \mathrm{O}_{2}, \mathrm{O}_{3}\right)$ :

$$
\begin{aligned}
& \forall\left(A_{1}, A_{2}, A_{3}\right) \in \mathcal{T}^{\mathcal{D} . A f f}, \\
& \left(A_{1}, A_{2}, A_{3}\right)+\left(O_{1}, O_{2}, O_{3}\right)= \\
& =\left(O_{1}, O_{2}, O_{3}\right)+\left(A_{1}, A_{2}, A_{3}\right)= \\
& =\left(A_{1}, A_{2}, A_{3}\right)
\end{aligned}
$$

As well as worth and below Propositions.

Proposition 3.4: Additions of three-vertexes is commutative in $\mathcal{T}_{\approx}^{\mathcal{D} \text {.Aff }}$ :

$$
\begin{aligned}
& \forall\left(A_{1}, A_{2}, A_{3}\right),\left(B_{1}, B_{2}, B_{3}\right) \in \mathcal{T}^{\mathcal{D} . A f f} \\
& \left(A_{1}, A_{2}, A_{3}\right)+\left(B_{1}, B_{2}, B_{3}\right)= \\
& =\left(B_{1}, B_{2}, B_{3}\right)+\left(A_{1}, A_{2}, A_{3}\right) .
\end{aligned}
$$

Proof: By definition of additions of three-vertexes that have:

$$
\left(A_{1}, A_{2}, A_{3}\right)+\left(B_{1}, B_{2}, B_{3}\right)=\left(A_{1}+B_{1}, A_{2}+B_{2}, A_{3}+B_{3}\right)
$$

From Theorem 2.1, in [3], have that for every two points is a line in the Desargues affine plane the addition is commutative, and consequently have to:

$$
\begin{aligned}
& \left(A_{1}, A_{2}, A_{3}\right)+\left(B_{1}, B_{2}, B_{3}\right)=\left(A_{1}+B_{1}, A_{2}+B_{2}, A_{3}+B_{3}\right) \\
& \stackrel{[1]}{=}\left(B_{1}+A_{1}, B_{2}+A_{2}, B_{3}+A_{3}\right)=\left(B_{1}, B_{2}, B_{3}\right)+\left(A_{1}, A_{2}, A_{3}\right) .
\end{aligned}
$$

Proposition 3.5: Addition of three-vertexes is associative in $\mathcal{T}_{\approx}^{\mathcal{D} . A f f}$ :

$$
\begin{aligned}
& \forall\left(A_{1}, A_{2}, A_{3}\right),\left(B_{1}, B_{2}, B_{3}\right),\left(C_{1}, C_{2}, C_{3}\right) \in \mathcal{T}_{\approx}^{\mathcal{D} . A f f} \\
& \left(A_{1}, A_{2}, A_{3}\right)+\left[\left(B_{1}, B_{2}, B_{3}\right)+\left(C_{1}, C_{2}, C_{3}\right)\right]= \\
& =\left[\left(A_{1}, A_{2}, A_{3}\right)+\left(B_{1}, B_{2}, B_{3}\right)\right]+\left(C_{1}, C_{2}, C_{3}\right)
\end{aligned}
$$

Proof: Let's have three whatever three-vertexes

$$
\left(A_{1}, A_{2}, A_{3}\right),\left(B_{1}, B_{2}, B_{3}\right),\left(C_{1}, C_{2}, C_{3}\right) \in \mathcal{T}_{\approx}^{\mathcal{D} . A f f}
$$

Appreciate now,

$$
\begin{aligned}
& \left(A_{1}, A_{2}, A_{3}\right)+\left[\left(B_{1}, B_{2}, B_{3}\right)+\left(C_{1}, C_{2}, C_{3}\right)\right]= \\
& =\left(A_{1}, A_{2}, A_{3}\right)+\left(B_{1}+C_{1}, B_{2}+C_{2}, B_{3}+C_{3}\right) \\
& =\left[A_{1}+\left(B_{1}+C_{1}\right), A_{2}+\left(B_{2}+C_{2}\right), A_{3}+\left(B_{3}+C_{3}\right)\right] \\
& \stackrel{[1]}{=}\left[\left(A_{1}+B_{1}\right)+C_{1},\left(A_{2}+B_{2}\right)+C_{2},\left(A_{3}+B_{3}\right)+C_{3}\right] \\
& =\left(A_{1}+B_{1}, A_{2}+B_{2}, A_{3}+B_{3}\right)+\left(C_{1}, C_{2}, C_{3}\right) \\
& =\left[\left(A_{1}, A_{2}, A_{3}\right)+\left(B_{1}, B_{2}, B_{3}\right)\right]+\left(C_{1}, C_{2}, C_{3}\right) .
\end{aligned}
$$

Proposition 3.6: For every three-vertex in $\mathcal{T}_{\approx}^{\mathcal{D} \text {.Aff }}$ exists her right symmetrical according to addition:

$$
\begin{gathered}
\forall\left(A_{1}, A_{2}, A_{3}\right) \in \mathcal{T}_{\approx}^{\mathcal{D} . A f f}, \exists \overline{\left(A_{1}, A_{2}, A_{3}\right)} \in \mathcal{T}_{\approx}^{\mathcal{D} . A f f}, \\
\left(A_{1}, A_{2}, A_{3}\right)+\overline{\left(A_{1}, A_{2}, A_{3}\right)}=\left(O_{1}, O_{2}, O_{3}\right)
\end{gathered}
$$

Proof: Let us have whatever $\left(A_{1}, A_{2}, A_{3}\right) \in \mathcal{T}_{\approx}^{\mathcal{D} . A f f}$, fix the 'zero' three-vertexes $\left(O_{1}, O_{2}, O_{3}\right) \in \mathcal{T}_{\approx}^{\mathcal{D} . A f f}$ (which would be similar to three-vertexes $\left.\left(A_{1}, A_{2}, A_{3}\right)\right)$ if apply the Proposition 3. 4, in [3] pp34990, have that, for points $\boldsymbol{A}_{1}, \boldsymbol{A}_{2}$ and $A_{3}$, find points respectively $\overline{A_{1}} \in O_{1} A_{1}, \overline{A_{2}} \in O_{2} A_{2}$ and $\overline{A_{3}} \in O_{3} A_{3}$ such that:

$$
A_{1}+\overline{A_{1}}=O_{1} ; A_{2}+\overline{A_{2}}=O_{2} ; A_{3}+\overline{A_{3}}=O_{3} .
$$

Well $\exists \overline{\left(A_{1}, A_{2}, A_{3}\right)}=\left(\overline{A_{1}}, \overline{A_{2}}, \overline{A_{3}}\right) \in \mathcal{T}_{\approx}^{\mathcal{D} . A f f}$ such that it: 


$$
\left(A_{1}, A_{2}, A_{3}\right)+\overline{\left(A_{1}, A_{2}, A_{3}\right)}=\left(O_{1}, O_{2}, O_{3}\right)
$$

I summarize what was said earlier in this

Theorem 3.2: The Groupoid $\left(\mathcal{T}_{\approx}^{\mathcal{D} . A f f},+\right)$ is commutative (abelian) Group.

\section{The Addition of Similarity $n$-Vertexes in the Desargues Affine Plane}

Equally as addition of three-vertexes in Desargues affine plane, by the same logic, additions and $n$-vertexes in this plane.

Remark 4.1: The set of similarity n-vertexes in Desargues affine plane marked with symbol $\mathcal{N}_{\approx}^{\mathcal{D} . A f f}$.

The addition algorithm of n-vertexes, by analogy with addition algorithm of the three-vertexes are presenting below:

Let's have two whatever similarity $n$-vertexes in Desargues affine plane:

$$
\left(A_{1}, A_{2}, A_{3}, \ldots, A_{n}\right),\left(B_{1}, B_{2}, B_{3}, \ldots, B_{n}\right) \in \mathcal{N}_{\approx}^{\mathcal{D} . A f f} .
$$

The definitions of the similarity of $\boldsymbol{n}$-vertexes have the following parallelisms:

$$
A_{1} A_{2}\left\|B_{1} B_{2}, A_{2} A_{3}\right\| B_{2} B_{3}, \ldots, A_{n-1} A_{n}\left\|B_{n-1} B_{n}, A_{n} A_{1}\right\| B_{n} B_{1}
$$

Constructed the lines $\boldsymbol{A}_{1} \boldsymbol{B}_{1}, \boldsymbol{A}_{2} \boldsymbol{B}_{2}, \boldsymbol{A}_{3} \boldsymbol{B}_{3}, \ldots, \boldsymbol{A}_{n} \boldsymbol{B}_{n}$, since are in Desargues affine plane, and from the parallels the above, are the conditions of the Desargues theorem, it results that the above lines or crossing from a fixed point $\mathbf{V}$ or they have a bunch of parallel lines.

In both cases equally found the zero $n$-vertex. Take one first point $O_{1} \in A_{1} B_{1}$, and then find all the other vertexes of n-vertexes how:

$$
O_{2}=A_{2} B_{2} \cap \ell_{A_{1} A_{2}}^{O_{1}}, O_{3}=A_{3} B_{3} \cap \ell_{A_{1} A_{3}}^{O_{1}}, \ldots, O_{n}=A_{n} B_{n} \cap \ell_{A_{1} A_{n}}^{O_{1}}
$$

Definition 4.1: In the above conditions, application

$$
+: \mathcal{N}_{\approx}^{\mathcal{D} \cdot A f f} \times \mathcal{N}_{\approx}^{\mathcal{D} . A f f} \rightarrow \mathcal{N}_{\approx}^{\mathcal{D} . A f f}
$$

defined by

$$
\begin{gathered}
{\left[\left(A_{1}, A_{2}, \ldots, A_{n}\right),\left(B_{1}, B_{2}, \ldots, B_{n}\right)\right] \mapsto\left(C_{1}, C_{2}, \ldots, C_{n}\right)} \\
\forall\left(A_{1}, A_{2}, A_{3}, \ldots, A_{\mathrm{n}}\right),\left(B_{1}, B_{2}, B_{3}, \ldots, B_{\mathrm{n}}\right) \in \mathcal{N}_{\approx}^{\mathcal{D} . A f f} \quad \text { call }
\end{gathered}
$$
the addition in $\mathcal{N}_{\approx}^{\mathcal{D} . A f f}$ according to this Definitioni, can write the addition algorithm of the $n$-vertexes:

$$
\forall\left(A_{1}, A_{2}, A_{3}, \ldots, A_{n}\right),\left(B_{1}, B_{2}, B_{3}, \ldots, B_{n}\right) \in \mathcal{N}_{\approx}^{\mathcal{D} . A f f}
$$

$$
\begin{aligned}
& \text { 1. } P_{1} \notin A_{1} B_{1}, A_{2} B_{2}, \ldots, A_{n} B_{n}, \\
& \text { 2. }\left[\begin{array}{l}
(i) \cdot \ell_{A_{1} B_{1}}^{P_{1}} \cap \ell_{O_{1} P_{1}}^{A_{1}}=P_{2}, \\
(i i) \cdot \ell_{P_{1} B_{1}}^{P_{2}} \cap A_{1} B_{1}=C_{1}
\end{array}\right] \\
& \text { 3. }\left[\begin{array}{l}
(i) \cdot \ell_{A_{2} B_{2}}^{P_{1}} \cap \ell_{O_{2} P_{1}}^{A_{2}}=P_{3}, \\
(i i) \cdot \ell_{P_{1} B_{2}}^{P_{3}} \cap A_{2} B_{2}=C_{2}
\end{array}\right] \\
& \text { 4. }\left[\begin{array}{l}
(i) \cdot \ell_{A_{3} B_{3}}^{P_{1}} \cap \ell_{O_{3} P_{1}}^{A_{3}}=P_{4}, \\
(i i) \cdot \ell_{P_{1} B_{3}}^{P_{3}} \cap A_{3} B_{3}=C_{3} . \\
\quad \vdots \\
\Leftrightarrow
\end{array}\right] \\
& \Leftrightarrow+1 .\left[\begin{array}{l}
(i) \cdot \ell_{A_{n} B_{n}}^{P_{1}} \cap \ell_{O_{n} P_{1}}^{A_{n}}=P_{n+1}, \\
(i i) \cdot \ell_{P_{1} B_{n}}^{P_{n+1}} \cap A_{n} B_{n}=C_{n} .
\end{array}\right] \\
& \left(A_{1}, A_{2}, \ldots, A_{n}\right)+\left(B_{1}, B_{2}, \ldots, B_{n}\right)=\left(C_{1}, C_{2}, \ldots, C_{n}\right) .
\end{aligned}
$$

And for $n$-vertexes, have true analog the statements had to three-vertexes (everything proved equally).

Well have the verities of following statements

Theorem 4.1: For every two n-vertexes $\left(A_{1}, A_{2}, \ldots, A_{n}\right)$, $\left(\boldsymbol{B}_{1}, \boldsymbol{B}_{2}, \ldots, \boldsymbol{B}_{n}\right) \in \mathcal{N}_{\approx}^{\mathcal{D} . A f f}$, algorithm (7) determines the single three-vertexes $\left(\boldsymbol{C}_{1}, \boldsymbol{C}_{2}, \ldots, \boldsymbol{C}_{n}\right) \in \mathcal{N}_{\approx}^{\mathcal{D} \text {.Aff }}$, which does not depend on the choice of hers auxiliary point $P_{1}$.

From Theorem 4.1, appears immediately true this

Proposition 4.1: Additions of $n$-vertexes in $\mathcal{N}_{\approx}^{\mathcal{D} . A f f}$ there are element zero the three-vertexes $\left(\boldsymbol{O}_{1}, \boldsymbol{O}_{2}, \ldots, \boldsymbol{O}_{n}\right)$ :

$$
\begin{aligned}
\forall\left(A_{1}, A_{2}, \ldots,\right. & \left.A_{n}\right) \in \mathcal{N}_{\approx}^{\mathcal{D} . A f f}, \exists\left(O_{1}, O_{2}, \ldots, O_{n}\right) \in \mathcal{N}_{\approx}^{\mathcal{D} . A f f} \\
& \left(A_{1}, A_{2}, \ldots, A_{n}\right)+\left(O_{1}, O_{2}, \ldots, O_{n}\right) \\
& \left(O_{1}, O_{2}, \ldots, O_{n}\right)+\left(A_{1}, A_{2}, \ldots, A_{n}\right) \\
& =\left(A_{1}, A_{2}, \ldots, A_{n}\right)
\end{aligned}
$$

Also well as worth and below Propositions.

Proposition 4.2: Additions of n-vertexes is commutative in $\mathcal{N}_{\approx}^{\mathcal{D} . A f f}$

$$
\begin{gathered}
\forall\left(A_{1}, A_{2}, \ldots, A_{n}\right),\left(B_{1}, B_{2}, \ldots, B_{n}\right) \in \mathcal{N}_{\approx}^{\mathcal{D} . A f f} \\
\quad\left(A_{1}, A_{2}, \ldots, A_{n}\right)+\left(B_{1}, B_{2}, \ldots, B_{n}\right) \\
=\left(B_{1}, B_{2}, \ldots, B_{n}\right)+\left(A_{1}, A_{2}, \ldots, A_{n}\right)
\end{gathered}
$$

Proposition 4.3: Addition of $n$-vertexes is associative in $\mathcal{N}_{\approx}^{\mathcal{D} . A f f}$

$$
\begin{aligned}
\forall & \left(A_{1}, A_{2}, \ldots, A_{n}\right),\left(B_{1}, B_{2}, \ldots, B_{n}\right),\left(C_{1}, C_{2}, \ldots, C_{n}\right) \in \mathcal{N}_{\approx}^{\mathcal{D} . A f f} \\
& \left(A_{1}, A_{2}, \ldots, A_{n}\right)+\left[\left(B_{1}, B_{2}, \ldots, B_{n}\right)+\left(C_{1}, C_{2}, \ldots, C_{n}\right)\right]= \\
= & {\left[\left(A_{1}, A_{2}, \ldots, A_{n}\right)+\left(B_{1}, B_{2}, \ldots, B_{n}\right)\right]+\left(C_{1}, C_{2}, \ldots, C_{n}\right) . }
\end{aligned}
$$




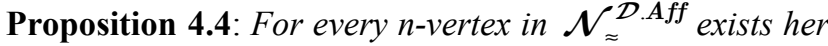
right symmetrical according to addition:

$$
\begin{gathered}
\forall\left(A_{1}, A_{2}, \ldots, A_{n}\right) \in \mathcal{N}_{\approx}^{\mathcal{D} . A f f}, \exists \overline{\left(A_{1}, A_{2}, \ldots, A_{n}\right)} \in \mathcal{N}_{\approx}^{\mathcal{D} . A f f} \\
\left(A_{1}, A_{2}, \ldots, A_{n}\right)+\overline{\left(A_{1}, A_{2}, \ldots, A_{n}\right)}=\left(O_{1}, O_{2}, \ldots, O_{n}\right)
\end{gathered}
$$

(Here have that $\overline{\left(A_{1}, A_{2}, \ldots, A_{n}\right)}=\left(\overline{A_{1}}, \overline{A_{2}}, \ldots, \overline{A_{n}}\right)$ )

By Theorem 4.1, Propositions 4.1, 4.2, 4.3 and 4.4 we have this true theorem:

Theorem 4.2: The Groupoid $\left(\mathcal{N}_{\approx}^{\mathcal{D} . A f f},+\right)$ is commutative (Abelian) Group.

\section{References}

[1] Dr. Orgest ZAKA, Prof. Dr. Kristaq FILIPI, (2017) An Application of Finite Affine Plane of Order $n$, in an Experiment Planning,"International Journal of Science and Research (IJSR),

http://www.ijsrpublications.com/ijsr.net/archive/v6i6/v6i6.php , Volume 6 Issue 6, June 2017, 1744 - 1747, DOI:

$10.21275 /$ ART20174592

[2] Zaka, O., Flipi, K. (2016). One construction of an affine plane over a corps. Journal of Advances in Mathematics, Council for Innovative Research. Volume 12 Number 5. 6200-6206.
[3] Zaka, O., Filipi, K. (2016). "The transform of a line of Desargues affine plane in an additive group of its points", International Journal of Current Research, 8, (07), 3498334990 .

[4] FRANCIS BORCEUX (2014). An Axiomatic Approach to Geometry\&An Algebraic Approach to Geometry (Geometric Trilogy I\& Geometric Trilogy II). Springer International Publishing Switzerland.

[5] GrRillet, P. A (2007). Abstract Algebra(Second Edition). Graduate Texts in Mathematics v242. ISBN-13: 978-0-38771567-4Springer Science + Business Media, LLC (Grrillet, P. A, et al, 2007).

[6] Hilbert. D, Vossen. S. C (1990). Geometry And The Imagination. Chelsea Publishing Company.

[7] Buenkenhout, F, (Editors)(1995). HANDBOOK OF INCIDENCE GEOMETRY. Elsevier Science B. V. ISBN: 0 $44488355 \mathrm{X}$.

[8] Hungerford, TH. W (1974). Algebra (Graduate Text in Mathematics vol 73). Springer-Verlag New York, Inc. ISBN 0387-90518-9.

[9] Lang, S (2002). Abstract Algebra (Third Edition). Springerverlag new york, inc. ISBN 0-387-95385-X.

[10] Ueberberg, Johannes (2011), Foundations of Incidence Geometry, Springer Monographs in Mathematics, Springer, doi:10.1007/978-3-642-20972-7, ISBN 978-3-642-26960-8. 